\title{
BioéthiqueOnline
}

\section{La mort de mon père}

\section{Jacques Quintin}

Volume 4, 2015

Reçu : 17 Dec 2014; publié : 8 Apr 2015; éditrices : Maude Laliberté \& Samia Cherfaoui

URI : https://id.erudit.org/iderudit/1035498ar

DOI : https://doi.org/10.7202/1035498ar

Aller au sommaire du numéro

Éditeur(s)

BioéthiqueOnline

ISSN

1923-2799 (numérique)

Découvrir la revue

Citer cet article

Quintin, J. (2015). La mort de mon père. BioéthiqueOnline, 4.

https://doi.org/10.7202/1035498ar
Résumé de l'article

Le texte suivant consiste en le récit de la fin de vie de mon père, à la maison sous les soins de ma mère, et illustre quelques enjeux éthiques, familiaux, de souffrance et de sens. Ce récit permet de montrer que la maladie et la mort, loin de se réduire à un problème à résoudre, deviennent un questionnement: qui sommes-nous, que devenons-nous? Plus que tout, il y a cette question : que pouvons-nous espérer de ces expériences de vie?
Ce document est protégé par la loi sur le droit d'auteur. L'utilisation des services d'Érudit (y compris la reproduction) est assujettie à sa politique d'utilisation que vous pouvez consulter en ligne.

https://apropos.erudit.org/fr/usagers/politique-dutilisation/ 


\title{
La mort de mon père
}

\author{
TRAVAIL CRÉATIF / CREATIVE WORK \\ Jacques Quintin ${ }^{1}$
}

Reçu/Received: 17 Dec 2014

Publié/Published: 8 Apr 2015

Éditrices/Editors: Maude Laliberté \& Samia Cherfaoui

2015 J Quintin, Creative Commons Attribution 4.0 International License

\begin{abstract}
Résumé
Le texte suivant consiste en le récit de la fin de vie de mon père, à la maison sous les soins de ma mère, et illustre quelques enjeux éthiques, familiaux, de souffrance et de sens. Ce récit permet de montrer que la maladie et la mort, loin de se réduire à un problème à résoudre, deviennent un questionnement: qui sommes-nous, que devenons-nous? Plus que tout, il y a cette question: que pouvons-nous espérer de ces expériences de vie?
\end{abstract}

Mots clés

Alzheimer, récit, éthique, mort, deuil

\begin{abstract}
Summary
The following text consists of the end of life story of my father, at home under the care of my mother, and illustrates some issues of ethics, family, suffering and meaning. This story helps to show that sickness and death, far from being reduced to a problem to solve, becomes a question: who are we and what do we become? And above all, there is the question: what can we expect from these life experiences?
\end{abstract}

\section{Keywords}

Alzheimer's, story, ethics, death, mourning

Affiliations des auteurs / Author Affiliations

${ }^{1}$ Faculté de médecine et des sciences de la santé, Bureau du développement de l'éthique, Université de Sherbrooke, Sherbrooke, Canada

\section{Correspondance / Correspondence}

Jacques Quintin, Jacques.Quintin@usherbrooke.ca

\section{Remerciements}

Ce récit se présente comme une forme de gratitude envers tous ceux et celles qui ont accompagné mon père dans son épreuve de vie.

Conflit d'intérêts

Aucun déclaré

\section{Acknowledgements}

This story is as a form of gratitude to all those who have accompanied my father in the challenges of life.

\section{Conflicts of Interest}

None to declare

«Quant à ma mort, elle ne sera fin racontée que dans le récit de ceux qui me survivront »

Ricœur

\section{Préambule}

Depuis le début des années 1980, j'ai exercé plusieurs fonctions dans le milieu hospitalier, principalement dans le milieu de la psychiatrie. Ceci n'est pas indifférent au déroulement des événements que vous lirez dans le texte suivant. Depuis 2006, je suis professeur d'éthique clinique à l'université de Sherbrooke. C'est dans ce contexte que ce texte trouve sa première motivation, et cela avant même le parcours de fin de vie de mon père. Je souhaitais écrire un texte à l'intention de mes étudiants qui illustrerait le fait que les personnes malades et les proches ne prennent pas leurs décisions strictement à la lumière des faits médicaux ni en prenant en compte les grands principes de la bioéthique que sont l'autonomie, la bienfaisance, la non-malfaisance et la justice, mais davantage selon ce qui fait du sens pour eux en raison de leur histoire de vie personnelle. De plus, je désirais aussi montrer qu'à travers le vécu des personnes impliquées dans une situation difficile ou de fin de vie émergent des questionnements ou des thèmes qui, loin d'être abstraits, deviennent partie intégrante de la réflexion éthique. La maladie, la souffrance, la mort, la solidarité, la justice, la tradition, l'identité personnelle en sont des exemples. 
Pour ces raisons, ce texte ne prétend pas donner une représentation exacte de ce qui a pu se passer. Au contraire, j'ai profité de l'occasion pour ajouter d'autres éléments plausibles (si peu), justement pour en rehausser la valeur pédagogique. Malgré tout, ce texte prend sa source dans un événement vécu de près qui m'a touché profondément, de sorte que ce texte en dit probablement davantage sur mon expérience (ou sur moi) que sur la mort de mon père, tel que vécu par lui-même ou les autres membres de la famille. Ensuite, il aurait été fastidieux de tout dire. Ce texte est donc à la jonction de l'autobiographie, de la fiction et de l'essai.

Ce texte se divise en deux parties : l'une où je relate les événements selon ma propre perspective et, l'autre, où je fais une brève analyse éthique. Cette deuxième partie, plus succincte, soulève bien d'autres enjeux que ceux nommés. II y aurait toute une réflexion à faire autour du fait que la situation de mon père diffère grandement des soins offerts en fin de vie à la population québécoise en général. Inutile de dire que peu de gens ont accès à des médecins spécialistes aussi facilement et en si grands nombres. La majorité va plutôt mourir à l'hôpital ou dans des établissements spécialisés dans des soins de fin de vie qu'à la maison. Sans compter l'énergie et la force de caractère de ma mère.

Même si j'ai modifié les noms de personnes concernées par la situation, elles ont toutes consenti au risque d'être reconnu.

\section{Introduction}

Mon père, René, a souffert de la maladie d'Alzheimer. Tout au long de la maladie, jusqu'à son décès et ses funérailles, différents enjeux éthiques, familiaux, de souffrances et de sens se sont posés pour chacun des membres de la famille selon leur propre histoire de vie. Dès les premiers signes alarmants de la maladie jusqu'à la fin de vie, s'insèrent dans l'existence de chacun des petits événements de vie, souvent perturbateurs, qui viennent chambouler la manière de comprendre ce que nous sommes comme être humain et le sens que nous accordons à l'existence. Prendre soin d'un proche, alors qu'on sait qu'il va nous quitter bientôt, c'est travailler sur soi.

C'est sur ce fond existentiel que les aidants naturels (épouse, enfants et autres) doivent faire des choix de vie pour la personne qui graduellement ne sera plus ce qu'elle était, quoique toujours là et en devenir. La maladie, loin de se réduire à un problème à résoudre, devient un questionnement : qui sommes-nous et que devenons-nous? Ces questions bouleversantes mettent l'identité de chacun à l'envers.

Cette expérience de la mort, pour ses témoins, conduit à une autre expérience peut-être plus fondamentale : celle de l'accomplissement de soi au travers de la culture de soi. Cela nous conduit à reconsidérer ce que signifie la culture. Comme toutes les bonnes histoires, le récit de la mort engendre à son tour de nouvelles expériences de vie qui interpellent d'autres récits et d'autres manières de se comprendre.

Je me permets d'introduire mon récit en me calquant sur ce que disait Descartes (1) à propos de son propre écrit le Discours de la méthode: "Cet écrit (n'est proposé) que comme une histoire, ou si vous l'aimez mieux, que comme une fable » dont le but n'est pas de décrire la réalité, mais de proposer un enseignement, voire une morale. Dans notre cas, la morale ou la leçon de cette histoire, on le verra, est multiple. Il est de mise de préciser qu'ici la morale n'a rien d'un ensemble de règles ou de maximes qu'il s'agirait de prescrire et de suivre. 


\section{Partie 1 : Récit des événements}

\section{Le temps de la maladie}

Avant que la maladie s'installe dans la vie de mon père et, ensuite, dans la vie de tous ceux qui l'entouraient, mon père n'a jamais été certain de la valeur de la vie. Quelque chose lui disait qu'il pourrait très bien ne pas exister et que cela ne ferait pas une grande différence. En raison de ce constat, il s'est toujours interrogé sur le sens de l'existence. Sa référence était la nature qu'il observait finement. II voyait en elle une leçon de vie : on naît, on se bat pour vivre, on meurt et rien d'autre. II était d'avis qu'il n'y avait pas de raison de se faire du souci outre mesure. Autant dire que mon père était un matérialiste. Pour cette raison, il disait souvent à ses enfants, lors de repas où l'on échangeait sur la vie, la spiritualité et la politique, que s'il devait devenir très malade, qu'on ne devrait pas insister à le garder en vie, mais qu'on devrait plutôt le laisser mourir et même précipiter sa mort. Ces mots étaient : " il n'y a rien là » et « on n'est que poussière ». Si mon père avait eu une connaissance des philosophes, il aurait partagé la pensée de Lucrèce, disciple d'Épicure, voulant que l'être humain, en l'occurrence son âme, n'est qu'un agrégat d'atomes qui se dissout au moment de la mort. C'est ma naissance, soit celle de son fils aîné, suivi de celle de ces deux filles, Louise et Catherine, et d'un autre garçon, André, qui a donné un sens à sa vie et donc une raison de la poursuivre.

Puis, est arrivé l'inévitable : la vieillesse. Par exemple, un samedi, j'ai demandé à mon père de venir m'aider à ériger une tonnelle au fond du terrain à une distance où nous n'avions pas accès à l'électricité même avec de longs câbles d'extension. Nous devions donc nous servir d'une génératrice. Toute la journée, mon père, le manuel, n'a montré aucun intérêt pour celle-ci et je devais donc lui expliquer, moi l'intellectuel, comment l'actionner. De plus, je devais constamment lui dire quoi faire pour m'aider. Vous devez savoir que mon père a travaillé toute sa vie dans la construction et, qu'en temps normal, nous nous serions disputés gentiment sur la meilleure façon de travailler. Tel n'était pas le cas ce matin-là. Sur l'heure du dîner, j'ai fait la remarque suivante à mon épouse : " je ne reconnais plus mon père ॥. J'ai mis cela sur le dos de la vieillesse. II avait 76 ans à l'époque. Après tout, ce sont des choses auxquelles on peut s'attendre. II n'y a pas de surprise majeure ou d'imprévus à cet égard. Lorsqu'on vieillit, c'est un peu dans l'ordre des choses que les personnes perdent de l'intérêt pour des activités ou des choses qui, jusqu'à tout récemment, les passionnaient. On nous le répète sans cesse. C'est un lieu commun d'entendre dire, à tort ou à raison, qu'en vieillissant on assiste à toutes sortes de pertes.

Dans les mois suivants, ma mère, Monique, a rapidement commencé à raconter un ensemble d'épisodes qui laissaient croire que quelque chose d'anormal se passait. Étant du milieu hospitalier, j'ai pu l'aider, avec tact, à réaliser que ce qu'elle me décrivait ressemblait beaucoup à la maladie d'Alzheimer.

J'ai donc demandé à Monique, qui avait commencé à accompagner René à ses rendez-vous médicaux, d'explorer la question avec le médecin. Après chaque rendez-vous, je lui demandais un compte rendu. À chaque fois, elle me disait que le médecin n'avait rien dit à ce sujet. Après deux ou trois rendez-vous qui ont abouti de la même manière, j'ai dit à mon père qu'à son prochain rendezvous, je l'accompagnerais. II m'a demandé quelles étaient les raisons sur un ton qui signifiait que je n'avais pas d'« affaire là ». Je l'ai donc accompagné à sa visite médicale malgré son refus initial.

Dans son bureau, le médecin a fait son entrevue selon les bonnes pratiques. J'ai senti que l'entrevue se terminerait bientôt. Alors, j'ai fait signe au médecin que si j'étais présent, c'était que j'avais des raisons d'y être. J'ai demandé à mon père de raconter ce qui se passait en faisant signe à Monique de ne pas intervenir. D'emblée, mon père a dit que ça n'allait pas, qu'il vivait des pertes de mémoire. Monique s'est mise à pleurer. Rapidement, le médecin a compris de quoi il était question. II a planifié une rencontre avec une gériatre exerçant en privé. Quelques jours après, le diagnostic est tombé. 
Même si mon père s'en doutait, même si Monique et moi n'avons pas été surpris, puisqu'on en avait déjà parlé, il n'en demeure pas moins que nous ne pouvions plus faire comme si de rien n'était. En fait, plus rien ne serait comme avant. À ce moment-là, les questions fusaient de toutes parts, autant pour mon père et pour Monique que pour tous leurs enfants. La principale question était la suivante : à quoi ressemblera l'avenir? Ce choc en est un dans la mesure où il renvoie à une autre question : que pouvons-nous faire? De surcroît, il reflète notre impuissance malgré notre responsabilité. Vient ensuite la question de notre identité. En effet, devant cette nouvelle réalité, une autre réalité s'installe aussi dans nos vies: on ne lit plus notre existence personnelle et familiale de la même façon. Commence alors une relecture de ce que nous sommes, qui nous amène à nous questionner au sujet de notre rapport à notre père, à notre mère et à la fratrie.

Si le diagnostic ne nous avait pas surpris, cependant, nous n'avions pas prévu que notre père puisse finir ces jours de cette manière. À partir de ce moment-là, c'est une suite d'imprévus qui s'installaient dans nos vies.

\section{Réactions face à la maladie}

$\mathrm{Si}$, pour mon père, plus rien ne va de soi et plus rien n'est comme avant, il en va de même pour nous tous. Des possibilités se ferment. Avant la mort de mon père, nous avons dû faire le deuil de bien des choses. Pas tant les choses que mon père ne pourra plus faire, mais le deuil d'une personne qui nous avait désirés, engendrés, nourris et aimés. C'est l'histoire de vie de chacun qui vient de basculer et, avec celle-ci, celle de notre identité. Encore une fois, plus rien ne sera pareil. Et rien de ceci n'était prévu.

En même temps, la question s'est posée ainsi : devons-nous faire comme si de rien n'était jusqu'au moment où cela ne sera plus possible? D'une certaine manière, notre réponse fut affirmative, même si nous avions commencé à modifier certaines choses. Je précise : "comme si de rien n'était » signifie pour nous que la vie continuerait comme avant sans cependant faire l'autruche ou instaurer des pratiques de négation. Ce n'est pas parce que cette maladie s'installe chez une personne et dans une famille qu'on doit cesser pour autant de vivre. D'autant plus que c'est une maladie qui s'étale sur plusieurs années. Donc, nous avons continué de vivre, mais en intégrant cette nouvelle réalité, tout en lui laissant un espace pour son expression.

Deux événements se sont produits peu de temps après la tombée du diagnostic. Le premier est en lien avec la réaction de Louise, ma sœur, qui habite aux États-Unis et qui croyait difficilement à la possibilité que notre père soit atteint de cette maladie. Elle nous prêtait de mauvaises intentions, comme celle de vouloir se débarrasser de lui. II y avait un lien très étroit entre Louise et René. Cependant, malgré ses visites régulières au Québec et leurs échanges téléphoniques hebdomadaires, il était clair pour moi que Louise n'a pas pu suivre l'évolution de la maladie de René au même rythme que nous. Elle n'avait pas le même vécu ni la même psychodynamique. Mon premier coup de génie, en raison de mon expertise dans le milieu médical, fut de proposer à Louise d'accueillir René chez elle en lui disant qu'il était possible que ce soit la dernière fois qu'il puisse le faire et qu'elle devait en profiter. Elle a accepté en reconnaissant que c'était une bonne idée. À sa première nuit chez elle, René s'est levé à $2 \mathrm{~h}$ de la nuit, nu dans le salon. Louise, plutôt étonnée, lui a demandé ce qu'il faisait là et elle a reçu comme réponse des propos insensés. Elle a immédiatement pu constater de visu de quoi il en retournait. À partir de ce moment, elle a accepté son diagnostic.

Monique est une femme d'affaires qui a tenu un commerce toute sa vie. Elle est une femme de très grande énergie. Par exemple, à 84 ans, elle joue encore au tennis sur une base quotidienne, s'adonne au ski alpin l'hiver et marche plus d'une heure par jour. Elle est également dotée d'une grande intelligence. C'est pour cette raison que j'ai dit à Louise que je l'encouragerais à aller chercher toute l'information possible sur la maladie d'Alzheimer à travers l'Association de la maladie d'Alzheimer, des sites Internet, et des livres, en croyant qu'elle s'investirait à fond dans cette démarche. C'est ce que j'ai fait. Ce fut le deuxième événement et mon deuxième coup de génie. 
Monique s'est effectivement engagée à fond dans cette nouvelle quête de connaissances. Cela lui a permis de mieux comprendre son mari, de faire preuve de plus d'affection et de douceur et de donner un sens nouveau à sa vie. Monique, comme moi d'ailleurs, a besoin de comprendre pour donner un sens à ses actions. À cet égard, nous avons des affinités avec la pensée de Platon, qui à travers Socrate, pensait que l'action juste, la vie morale ou la vertu s'appuient sur la connaissance. Mon intuition était que Monique deviendrait une soignante de première classe très dévouée.

Ensuite, Monique est une personne qui aime relever les défis. Dans sa tête, elle se présente comme la femme parfaite, le super héros, la femme forte, celle qui porte le monde sur ses épaules. Sans connaître la pensée de Kant et son ouvrage Fondements de la métaphysique des mœurs (2), pour qui la morale se réduit au respect de l'impératif catégorique, Monique a tout fait par devoir moral. Elle a aussi tout fait pour être à la hauteur de son idéal de perfection : être irréprochable. Bref, nous avons tous profité de son engagement.

Elle est également croyante, pratiquante et engagée dans des activités de bénévolat au sein de la paroisse. Cependant, je ne suis pas en mesure de faire ressortir à quel point cela a pu devenir un facteur pour son dévouement. Certes, le sacrifice de soi, le désir de gagner son ciel et faire preuve de charité sont des composantes sûrement essentielles à sa démarche. Cela me fait réaliser à quel point je n'ai jamais eu de discussions réelles et sérieuses sur le thème de la vie spirituelle.

Rapidement, par déformation professionnelle, j'ai encouragé Monique à préparer le placement éventuellement de mon père dans un établissement privé, bien apprécié dans le milieu, en inscrivant son nom sur la liste d'attente. Je lui ai expliqué que ce ne sera peut-être que dans deux, voire trois ans, mais que la situation deviendrait de plus en plus difficile pour elle et qu'elle n'était pas tenue d'accomplir l'impossible. Tout aussi rapidement, j'ai compris que Monique avait la ferme intention de prendre soin de mon père à la maison jusqu'à la fin de sa vie ou presque. Étant une femme de raison et raisonnable, elle n'aurait pas été fermée à l'idée d'un placement en toute fin de vie, justement en raison des conditions médicales et des soins requis. Cependant, j'ai senti que son idée était faite et que cela donnerait un sens nouveau à sa vie, ou plutôt, que cela s'inscrivait très bien dans son histoire de vie et dans ses valeurs. Elle croyait aussi que mon père n'aurait pas apprécié d'être placé; elle avait tout à fait raison.

Lorsque mon père a reçu son diagnostic, il a voulu mettre fin à ses jours. II en parlait ouvertement à tous les membres de la famille. Exerçant dans le domaine de la psychiatrie, on s'est évidemment tourné vers moi pour savoir ce que j'en pensais, et surtout quoi faire. Ce fut très difficile pour moi. La difficulté était de pouvoir en parler de manière très rationnelle, comme mon père le faisait lui-même. Par conséquent, j'ai décidé d'adopter, autant que possible, une posture professionnelle, c'est-à-dire de ne pas l'influencer, de lui signifier, peu importe son choix, qu'on vivrait avec cela, mais de s'assurer que celui-ci soit bien réfléchi. Nous avons eu plusieurs discussions, toutes marquées par l'ouverture et le respect. Un jour sans que je lui demande où il en était rendu dans sa réflexion, il m'a répondu que tout compte fait, il ne mettrait pas fin à ses jours. Sa justification était qu'il ne voulait pas nous faire vivre cela. Encore une fois, il sacrifierait son premier choix pour nous. Autrement dit, il nous a choisis avant de se choisir, ou de penser seulement à lui. Il disait aussi qu'après tout, Monique, son épouse, quoique brusque parfois, faisait un travail extraordinaire. Cela l'étonnait qu'on veuille et qu'on prenne autant soin de lui. II en a toujours été très reconnaissant. Si c'est la naissance de ses enfants qui lui a permis de donner un sens à sa vie, c'est en retour, le soin, le souci et le lien de ses enfants qui maintenant donnaient un sens à sa vie sans compter l'engagement de son épouse.

Depuis le début de la maladie, ma mère, mon frère, mes sœurs et moi, nous ne nous sommes jamais autant parlé. Ce n'était pas toujours facile, dans la mesure où chacun d'entre nous avait non seulement sa perception et sa propre histoire avec mon père, mais en avait aussi avec Monique, sans compter nos histoires entre nous. Par contre, il n'y a pas eu de disputes. Au contraire, dès le départ 
ce sont de multiples consensus qui se sont établis au fur et à mesure que la maladie avançait, sans compter une nouvelle solidarité.

D'un point de vue phénoménologique, la maladie est décrite comme une expérience dans laquelle le temps se rétrécit au moment présent. La maladie se vit comme un appauvrissement du temps. Le monde se ferme sur lui-même. II y a de moins en moins de possibilités. Tout s'inscrit dans un présent qui n'en finit plus de durer, sans ouverture sur le passé et l'avenir. La maladie s'acharne et écrase la personne malade. Ce faisant, même si nous avions décidé de continuer de vivre dans la transparence, nous vivions la maladie de l'autre. Nous revivions ce rétrécissement du temps, du possible. Tout se fermait autour de nous. La vie s'arrêtait avec la maladie du malade. Dans un tel contexte, on pourrait se demander qui meurt? À travers la mort qui s'emparait de mon père, c'est tous ceux qui se sentaient interpellés par sa condition qui vivaient, à leur manière, cette mort. II y a quelque chose en nous qui ne sera plus jamais comme avant. C'est comme si un nouvel élément venait s'introduire, par la force des choses, dans le récit de nos vies et cela, ce n'était pas prévu, tout comme la maladie d'Alzheimer de mon père.

Nous avons constamment encouragé Monique à maintenir ses activités de bénévolat (eh oui! du bénévolat comme si elle n'en faisait déjà pas assez), qui consistait à aider les plus démunis à effectuer leur rapport annuel de revenu, ainsi que ses activités sportives, comme le tennis et le ski. Ceci impliquait qu'une de mes sœurs, Catherine, en raison de la proximité de son domicile et de sa disponibilité, vienne garder René à la maison durant ces moments. La sœur de René, Pierrette, était également présente durant cette difficile période. Celle-ci le considérait comme son frère préféré sur une fratrie de 5 garçons, car il était le seul qui avait manifesté un réel souci pour elle lorsqu'elle était plus jeune, encore à la maison en train de faire de multiples travaux domestiques. Régulièrement, il lui avait proposé de l'aider. Elle s'en est toujours souvenue.

Ce souci de fournir du répit à Monique impliquait aussi tous les enfants, selon leurs disponibilités, souvent réduites en raison de la distance géographique qui nous séparait de la maison familiale. Avec le temps, des bénévoles provenant du CLSC, qui offre des soins à domicile, se sont ajoutés, même si cela n'était pas toujours aidant pour nous. Par exemple, recevoir un bénévole le lundi matin à $8 \mathrm{~h}$ ne permettait pas à ma mère d'effectuer son magasinage ou de s'occuper de tout ce que le quotidien exige. Tous les commerces sont fermés à cette heure-là. Dans la dernière année de sa vie, lorsque mon père fut de plus en plus en perte d'autonomie, la même aide à la même heure fut cependant utile pour les bains, car ils étaient devenus trop exigeants physiquement pour Monique, malgré sa grande force physique.

D'un point de vue extérieur, l'aide des bénévoles provenant du CLSC peut sembler être un gain, mais il faut comprendre que le fait d'accepter que des étrangers entrent dans sa maison et s'y installent d'une certaine façon n'est pas une chose facile pour Monique. Ensuite, elle a dû plier sur son orgueil afin de reconnaître le fait qu'elle ait besoin d'aide, elle qui se présente comme une superwoman. Encore une fois, son côté raisonnable a pris le dessus.

Cependant, je crois que le plus difficile pour tous les membres de ma famille était la solitude, ou plus précisément le fait de constater combien Monique était seule, de savoir à quel point elle se sentait seule dans cette aventure qui n'en finissait plus malgré notre présence et nos visites assidues. Même si elle a pu poursuivre ses quelques activités sportives, il y a une lourdeur existentielle difficilement partageable qui s'installe. Même si on en parlait, il n'en demeure pas moins que de le dire et de le vivre sont deux réalités d'ordre de grandeur différentes.

En plus, le dévouement extrême de Monique a mis en évidence les limites de ses capacités, jusqu'ici considérées comme hors normes. Nous observions tous le fait que Monique montrait de plus en plus de signes de fatigue. Comme l'expression le dit, elle avait pris un coup de vieux. Durant ces huit années, Monique n'a cessé de vieillir. Nous la surprenions à dormir régulièrement en plein après-midi 
sur sa chaise en notre présence. Elle était épuisée. Nous n'avions jamais vu Monique dormir durant le jour ni être malade de quoi que ce soit, même pas un rhume. Cela suscitait une énorme crainte pour nous : et si elle mourait avant notre père, d'un infarctus par exemple? Nous nous sentions terrorisés et impuissants face à cette éventualité de perdre notre mère avant notre père. Surtout qu'il n'était pas question de remettre en doute le projet de vie de Monique : prendre soin de son époux. De 76 ans à 84 ans, il se passe bien des choses dans la physiologie humaine.

Malgré toutes les connaissances factuelles et le soutien technique, le plus difficile est de ne jamais savoir ce qui arrivera. C'est l'appréhension constante de ce que le futur nous réserve. Nous pouvons toujours gérer le présent, mais le futur demeure imprévisible. Comment vivrons-nous le nouvel état de René? Le plus difficile c'est l'imprévisible et l'incontrôlable. II s'agit d'une expérience qui nous amène constamment ailleurs et, contrairement aux autres expériences de notre vie, celle-ci n'était pas prévue.

\section{L'épisode du décès}

Avec le décès tout redevient possible, cependant, sans jamais redevenir comme avant : il y a des choses qu'on ne peut plus récupérer. On n'est plus la même personne après une telle expérience. Les possibilités ne sont plus les mêmes. La vie n'a plus le même sens. Cela aussi n'était pas prévu.

Sept jours avant le décès de mon père, Louise qui habite aux États-Unis, à la suite d'une conversation téléphonique avec Monique, a senti une urgence non négociable de venir l'épauler à la maison familiale. Deux jours après son arrivée, mon père a commencé à se plaindre de douleur. II ne parvenait plus à s'alimenter ni à s'hydrater malgré l'aide fournie. Étant inquiète et ne sachant pas quoi faire, elle a décidé d'appeler l'un de nos cousins, Paul, qui est médecin, en l'occurrence un spécialiste en soins de fin de vie. Comme s'il n'y a pas de hasard dans la vie, il s'avère qu'il était en vacances pour une semaine et qu'il n'avait rien de prévu. II a dit ce qu'il en pensait et a indiqué qu'il sera présent dès le lendemain, soit le lundi.

À son arrivée, il a établi un pronostic selon lequel mon père décèdera probablement à l'intérieur des cinq prochaines journées. Avec l'accord de Monique, il a amorcé les administrations de morphine pour soulager sa douleur. II nous a exposé ce à quoi on devait s'attendre pour les prochains jours en nous expliquant les symptômes et les signes qui apparaîtront. II est demeuré au chevet de mon père les 72 heures suivantes. Inutile de vous dire que sa présence a fait toute la différence entre une expérience positive et négative de la fin de vie. Inutile de vous dire qu'en raison du dévouement et de l'énergie de Monique, de la présence de quelques bénévoles, d'un service privé de gériatrie et d'un médecin au chevet dans les derniers instants, la question de l'euthanasie ne s'est pas posée. Certes, il existe des conditions pathologiques qui ne sont pas reluisantes et qui sont difficiles à vivre. Ma mère, mon frère, mes sœurs et moi avons tous reconnu que nous avons été privilégiés. Même si la maladie est une expérience difficile, voire inhumaine, l'environnement dans lequel se déploie la maladie peut devenir humanisant. Ce qui est probablement encore plus significatif dans notre cas, ce fut l'environnement qui a permis à chacun de nous de prendre la parole.

À partir du moment où la fin est apparue inévitable et dans un avenir rapproché, le discours de tout un chacun a changé d'une manière remarquable. Comme l'indique le philosophe Pierre Hadot (3), " le langage ne se développe que sur la mort des individus ". Nous nous disions que René, même en sédation, était probablement heureux que nous soyons tous là réunis autour de lui. Dès que nous étions tous présents à la maison et que mon cousin a confirmé que c'était terminé, Monique s'est montrée soulagée, comme si on venait de lui enlever un immense poids sur les épaules. Enfin, il se passait quelque chose. II faut comprendre que la maladie de l'Alzheimer induit des changements si peu perceptibles, que c'est comme s'il n'y avait jamais rien qui se passait. C'est d'un ennui mortel. 


\section{Partie 2 : Analyse éthique}

\section{Le besoin de raconter}

À l'intérieur d'une démarche herméneutique, il est de mise de se demander ce qui peut bien ressortir de cette histoire. À quoi ce récit renvoie-t-il? À quelles idées? Bref, à quoi ce récit nous fait-il penser? Que pouvons-nous retirer de cette expérience qui s'est étendue sur huit années? Ou encore, qu'estce que cela a changé pour chacun de nous?

La réponse est toute simple. Concrètement, à l'exception du quotidien de Monique, il n'y a pas eu de changement majeur. Par contre, il y a eu une foule de petits changements qui se sont déroulés dans notre existence psychique, qui n'étaient pas prévus et qui nous surprenaient. Ce que cette expérience a produit, c'est, en outre, une amplification de nos pensées, de nos sentiments, de nos réflexions et de nos valeurs. Ce qui souvent demeurait implicite devenait maintenant plus explicite. Grosso modo, c'est notre compréhension de la réalité qui s'est modifiée, ainsi que notre compréhension de qui nous sommes les uns envers les autres.

Cela a aussi mis en évidence que, lorsque Monique décèdera à son tour, les liens de la fratrie se dissoudront. Chacun poursuivra sa route, sa destinée. Cela ne signifie pas que l'on ne se rencontrera pas, mais que les occasions se feront plus rares. Nous l'avons vécu avec nos grands-parents. Nous le vivrons maintenant avec nos parents. Avec la mort, il n'y a plus rien de pareil. Cela induit des deuils, un brin de nostalgie. Autrement dit, avec la mort de mon père, c'était un peu comme la mort de la famille. Ce qui nous unit maintenant, certes il y a encore Monique, c'est ce que nous avons bâti ensemble et ce que nous comptons faire ensemble à l'avenir. Ce n'est plus la famille qui nous unira. Ce sera ce que nous sommes à la lumière de notre passé, mais surtout à la lumière de ce que nous pensons devenir.

C'est pour cette raison qu'à la suite des funérailles de mon père, entre cousins et cousines, nous avons convenu ensemble d'organiser une journée de rassemblement incluant les grands-parents, les parents, les enfants et tous les petits-enfants. Il y avait un besoin de se rencontrer et de se raconter ce que nous avons vécu ensemble lorsque nous étions plus jeunes lors des rassemblements de famille presque hebdomadaires, tout en sachant très bien que chacun de nous était engagé dans des trajectoires de vie tout à fait singulières. La journée était prévue en septembre 2014. Les tantes et les oncles nous ont rappelé de faire vite, car il se pouvait très bien que d'ici là d'autres nous quittent. Évidemment, statistiquement, lorsqu'on sait qu'ils sont tous âgés de 75 et 95 ans, cela est effectivement très probable. D'ailleurs, un oncle nous a quittés depuis, en août 2013. II n'en reste que sept, dont un, le plus jeune du groupe, qui a reçu dernièrement le diagnostic d'Alzheimer et un autre qui a été placé dans un centre hospitalier de soins de longue durée. Ce n'est pas seulement une génération entière qui nous quitte, mais tout un monde, difficilement compréhensible, pour ne pas dire complètement incompréhensible, pour les plus jeunes.

Exemple à l'appui, dernièrement, l'une de mes cousines nous racontait que son père avait reçu une vache en cadeau de son père (en l'occurrence mon grand-père) afin de préparer son avenir et que celui-ci l'avait vendue quelques années plus tard. Cela s'est déroulé à la fin des années cinquante. Cet oncle a par la suite connu du succès dans les affaires. Lorsque ma cousine avait raconté cette histoire à son fils de 10 ans, celui-ci ne comprenait pas et il ne comprend toujours pas aujourd'hui à 22 ans ce que c'est que de recevoir une vache en cadeau. Certes, il sait très bien ce qu'est une vache, mais n'arrive toujours pas à comprendre le monde vécu de cette période de vie, c'est-à-dire tout ce qu'une vache peut représenter pour des gens à cette époque pas si lointaine.

Mes oncles, comme mon père, étaient de grands raconteurs. Ce sont donc tous leurs récits qui nous quittent avec eux. D'un autre côté, avec le décès, ce sont d'autres récits qui s'installent et qui prennent forme. Au salon funéraire, souvent les échanges commençaient comme suit: "Te souviens-tu? »Cela étant dit, s'il y a quelque chose qui ne meurt pas c'est le besoin de raconter ce 
que l'on a vécu comme si nous voulions perpétuer quelque chose. Les Grecs anciens disaient que la gloire consistait à passer à l'histoire, à ce qu'on se souvienne de soi parce qu'on aurait changé le cours des choses. Si l'histoire existe, c'est parce qu'il y a eu des poètes et des gens qui ont érigé des monuments commémoratifs. De plus, ces histoires ont le don de créer ce dont elles parlent. Ce sont ces histoires qui donnent accès à l'immortalité. Les hommes meurent, les paroles demeurent. Ce qui survit, c'est le récit qu'on peut faire de la personne décédée. Je me souviendrai de mon père et de cette expérience de la maladie et de la mort selon le récit que j'en ferai. La mémoire se constitue à travers la mise en récit.

Pour cette raison, lors des funérailles à l'église, j'ai partagé, devant les gens réunis, ma propre histoire de mon propre père. La voici.

\section{L'oraison funèbre}

Jean-Jacques Rousseau (4), un penseur du XVIII siècle, disait: "L'homme ne vit qu'à moitié durant sa vie, et la vie de l'âme ne commence qu'à la mort du corps ". On dit qu'il est impossible de faire l'expérience de sa propre mort, parce que mourir, c'est ne plus faire d'expériences. II semble que la maladie de l'Alzheimer montre le contraire.

Longtemps avant de mourir, René a fait l'expérience de la mort. II a vu ce que c'était de mourir, tranquillement. II a fait l'expérience du détachement extrême. Dans cette expérience, il ne lui restait plus que la vie dans toute sa nudité, dans sa plus grande simplicité, dans sa plus grande candeur, dans toute son affectivité. II ne restait plus que l'amour.

René est décédé. Lorsqu'une personne meurt, une personne qu'on connaissait et qu'on aimait, on se demande toujours un petit peu qui était cette personne. Qui était René? II y a autant de René qu'il y a eu de personnes qui l'ont connu. II y a eu le René de sa mère, Agathe, et de son père, Eugène. Le René de ses tantes, de ses oncles, de ses cousins et de ses cousines. II y a eu le René de ses amis. II y a eu le René de ses frères: Marcel, Gaston, Daniel et Raymond. II y a eu le René de sa sœur, Pierrette. II y a eu le René de ses belles-sœurs et de ses beaux-frères. II y a eu le René de ses neveux et nièces. Il y a eu le René de son épouse Monique. Le René de ses enfants : Louise, Catherine, André et moi-même. Il y a eu aussi le René de ses brus et gendres: Lucie, Nicole, Antoine, et Luc. II y eut le René de ses petits-enfants : Mélanie, Marjorie, Isabelle, Claudia, Charlène, Jérémie et Matthieu. Ça fait bien des René. Tous ont quelque chose à dire à propos de lui. Mais la question demeure : qui était René?

Pour comprendre, il n'y a rien de mieux que de raconter une histoire. Une histoire vraie. Je vais donc vous dire qui était René pour moi. Je vais donc vous raconter deux petites histoires. La première s'est déroulée il y a plusieurs années déjà. J'avais 11 ans, c'était une belle journée, le 28 septembre 1968. Une journée ensoleillée et chaude. C'était un samedi. René venait de terminer sa journée de travail, car à cette époque, il travaillait le samedi. II était midi, on dînait et je lui ai demandé si nous pouvions aller à la chasse à la perdrix. II m'a répondu qu'on ne pouvait pas y aller parce que ce n'était pas encore le temps, que c'était trop tôt, qu'il y avait encore trop de feuilles dans les arbres, qu'on ne verrait rien. En plus, il disait qu'il faisait trop chaud, que les perdrix ne bougeraient d'aucune façon. J'étais bien déçu, mais j'aimais tellement ça, et comme un petit garçon peut le faire à cet âge, j'ai beaucoup insisté, j'ai sorti tous les arguments possibles pour faire changer d'idée à René. J'ai réussi.

Nous sommes donc allés chez Eugène, sur ses terres. Nous avons parcouru toutes les pointes de bois. Évidemment, René avait raison. Aucune perdrix en vue. J'étais bien déçu à l'idée de revenir bredouille. À notre retour, il m'avise qu'on pourrait faire le guet plutôt que de traquer le gibier. Lorsque nous sommes arrivés à un lieu stratégique, il m'invite à m'asseoir tranquillement sur un tronc d'arbre étendu par terre et à ne pas bouger ni à parler. René fait de même en s'appuyant contre un arbre. Et là, il s'est passé quelque chose d'extraordinaire. Après deux minutes, c'est long pour un enfant deux minutes à ne rien faire, j'ai commencé à ressentir une sorte de trouille. J'ai dû attendre environ une 
dizaine de minutes à écouter le silence de la nature, à écouter la légère brise dans les arbres. J'ai ressenti une grande angoisse. J'ai ressenti toute la puissance de la nature. La nature, ça parle. En même temps, j'ai ressenti toute la puissance de ma propre présence. II y avait quelque chose qui existait en moi.

Une dizaine de minutes plus tard, René se tourne vers moi, me fait signe encore une fois de ne pas bouger ni de parler, se lève, se retourne, lève son fusil, pointe et tire. Je ne comprenais pas trop, mais en même temps je comprenais. II me dit alors que je pouvais aller chercher la perdrix qui reposait à quelques pieds à peine d'où nous étions. J'étais ravi. Nous n'étions pas venus pour rien.

Si je vous raconte cette histoire, ce n'est pas pour vous raconter une histoire de chasse, mais c'est pour vous raconter qui était René. En effet, cette expérience de chasse m'a fait découvrir qui était mon père. J'ai compris qu'il était mû par le désir de comprendre la Nature. II était convaincu qu'elle était remplie de sagesse et de vérité. On n'avait qu'à observer pour en tirer un enseignement, une leçon de vie : apprendre à mourir. D'ailleurs, il me disait souvent que la vie était bien faite. On naît, on se développe, et on meurt. Ainsi va la vie. Dans cette vision des choses, la mort n'a rien de terrible. La mort c'est la vie. II n'y a rien de plus naturel que de mourir. C'est un phénomène de vie d'une grande valeur. Bref, René était un philosophe. II désirait et aimait la sagesse. J'en suis son héritier.

Le désir de sagesse, René l'exprimait aussi dans sa manière d'accueillir les gens. Il aimait les gens. Sa grande force était le don qu'il avait de les " faire parler ". II avait le don, comme Socrate, d'aider les gens à approfondir leur pensée sans jamais les confronter, tout simplement en amenant un autre point de vue, une nuance, une écoute attentive. II essayait toujours de comprendre. II était convaincu que les gens avaient en eux une partie de la vérité : autant les gens près de lui que les gens dans les contrées lointaines, pauvres ou riches. II incarnait l'hospitalité. C'est la raison pour laquelle René était fasciné par les émissions de télévision à propos du monde animal, des autres peuples et des autres sociétés. Nous visionnions ensemble les Grands Explorateurs.

Ceci m'amène à vous raconter ma deuxième histoire, plus courte, mais qui fait le lien avec la première. C'était à l'époque où Louise et moi fréquentions le Cegep. C'était dans les années 1970 avec toute l'effervescence de ces années remplies de remises en question. À la maison, durant le souper nous discourions souvent de politique, de société et de révolution. Nos discussions se continuaient longtemps après le repas. Nous refaisions le monde ensemble. Un jour, durant l'une de ces discussions épiques, mon père se tourne vers moi et nous dit que nous devions écrire et publier ce que nous pensions pour changer le monde. Je ne sais pas pourquoi, mais lorsqu'il a prononcé ces paroles, celles-ci ont résonné en moi. Je n'ai jamais oublié.

Pourquoi ai-je choisi ces deux histoires? C'est pour vous dire qui était René pour moi. C'est aussi pour vous dire que ces deux histoires illustrent l'héritage que j'ai reçu de sa part très tôt dans ma vie, soit comprendre la vie et la rendre meilleure pour les êtres humains. Depuis, j'exerce le métier de philosophe et j'écris. Cet héritage vaut une fortune pour moi. J'aimerais maintenant le transmettre à mon fils Jérémie, qui saura ensuite le transmettre aussi, à sa manière, aux générations futures.

René cherchait la sagesse et la vérité. Je ne sais pas s'il les a trouvées, mais une chose est certaine, il a trouvé dans ses enfants un sens à sa vie. Je peux donc dire : mission accomplie. II s'est acquitté de son devoir de façon admirable. Lorsqu'on a fait ses devoirs, on peut quitter cette vie en paix. De la même façon que René se disait « good job » lorsqu'il terminait sa journée de travail et qu'il constatait le nombre de verges qu'il avait creusées avec sa pelle mécanique, je peux maintenant dire : "Papa, good job. Grâce à toi, nous, tes enfants, sommes tous fiers de ce que nous sommes devenus ».

René était très humble. J'ai bien dit qu'il cherchait la sagesse. Je n'ai jamais dit qu'il l'avait trouvée. Peu importe ce qu'il pensait, il ne se prenait jamais au sérieux. II avait un sens de la dérision très affiné. Cette dérision, nous l'avons vécue avec lui jusqu'à la fin. Cela fait aussi partie de notre 
héritage. II était si humble que, s'il m'entendait discourir ainsi, il dirait « tu parles trop fiston ». Mais pour devenir un digne héritier de René, j'ai dû fréquemment faire à ma tête. J'ai dû penser par moimême et je continuerai, pour honorer sa mémoire.

Dans toute son humilité, il remercierait son épouse de s'être sacrifiée durant toutes ces années pour lui. II aurait dit que cela ne valait pas le trouble, mais lui remettrait quand même une digne mention d'honneur: "Merci Monique pour toute l'excellence que tu as mise dans tes soins. Good job ». II dirait aussi : "Merci Catherine pour ta disponibilité. Merci Louise pour ta qualité de présence et tes bons mots. Merci André pour le souci de mon bien-être ". II dirait aussi merci à sa cousine Marcelle de la manière suivante : "Ta présence était un baume de douceur sur ma mémoire écorchée ». II remercierait aussi son neveu Paul, médecin, pour son expertise et son dévouement en lui disant : "Ce fut grandement apprécié. Ce fut inestimable ». II dirait aussi merci à sa sœur Pierrette pour son accompagnement et ses petites douceurs. II vous dirait à vous tous, ici présents : "Merci d'être là, même si ce n'était pas nécessaire de se donner tout ce trouble ».

Pour René, il y avait très peu de choses nécessaires dans la vie. C'est un homme de simplicité. Manger, travailler, dormir et converser avec les gens autour de lui, lui suffisaient. II n'avait jamais lu Épicure, pourtant c'était un épicurien, non pas au sens d'un homme de plaisir, mais celui d'un homme qui savait régler sa vie en regard des besoins essentiels. C'était aussi un sceptique, un grand sceptique. II se méfiait de tout ce qui pouvait l'éloigner de ce qu'il considérait être la vraie vie.

Aristote (5), un philosophe qui a vécu au troisième siècle av. J.-C., disait qu'on ne pouvait pas juger la vie d'un homme avant que celle-ci ne soit terminée. Maintenant que tout est terminé, il peut dire : "Tout compte fait, la vie a été bonne pour moi. Même ma maladie, grâce à Monique, ne fut pas si terrible. Même ma mort, grâce à Paul, ne fut pas si atroce ».

René a dit: "sic vitam est. Vita brevis, ars vitae longa, tempus non conficiat fatuitatibus ». Je traduis: "Ainsi va la vie. La vie est brève, l'art de vivre long. Ne perdez pas votre temps à des niaiseries ». Merci.

\section{La morale de ce récit}

Descartes (1) disait la chose suivante : " même les histoires les plus fidèles, si elles ne changent ni n'augmentent la valeur des choses pour les rendre plus dignes d'être lues, au moins en omettentelles presque toujours les plus basses et moins illustres circonstances, d'où vient que le reste ne paraît pas tel qu'il est ». Évidemment, ce portrait de mon père est un portrait idéalisé. Par le terme " idéalisé », je n'entends pas « embelli », mais plutôt l'idée que je m'en fais. Alberto Manguel (6) dit la chose suivante :

Les histoires [...] sont notre façon de rendre compte de notre expérience du monde, de nous-mêmes et des autres [...] Dans certains cas, les histoires peuvent nous venir en aide. Elles peuvent parfois nous guérir, nous illuminer et nous montrer le chemin. Et, surtout, elles peuvent nous rappeler notre condition, percer l'apparence superficielle des choses et susciter en nous l'intuition des courants et des profondeurs sousjacents. Les histoires peuvent alimenter notre conscience et, par là, nous amener à savoir sinon qui nous sommes, du moins que nous sommes, connaissance essentielle qui s'enrichit par la confrontation avec la voix d'autrui.

Cette citation résume la morale de cette histoire, soit que l'expérience de la mort vient amplifier notre compréhension de la condition humaine et celle de notre rapport à l'existence. La mort met en dialogue ceux qui restent. Elle met aussi en dialogue le sens de la vie personnelle de chacun avec le sens de la vie générale. Cette question du sens surgit de la vie elle-même qui vient poser une distance avec la vie. C'est une expérience qui vient grossir un regard qui met en avant-scène des choses restées jusque-là dans l'ombre. Quelques mois après la mort de mon père, je peux affirmer 
que je me retrouve avec une compréhension plus affinée du sens de l'existence ainsi qu'une urgence de vivre et d'accomplir ce que je pense devoir faire. Cette compréhension s'accompagne d'une nouvelle hiérarchisation des valeurs, des désirs et des besoins. Elle s'accompagne également d'un sentiment de non-peur de la mort, un sentiment d'être en paix avec la vie et avec les gens. Enfin, elle s'accompagne d'une plus grande tolérance aux parcours de vie de chacun comme si chacun avait à vivre sa propre histoire avec le sentiment que tous ces parcours de vie auront une fin.

La mort d'un être cher nous rappelle notre devoir de continuer à vivre, à faire ce que nous devons faire, à poursuivre notre accomplissement, à faire naître quelque chose autour de soi en faisant des choix, c'est-à-dire en laissant mourir des options. Elle nous rappelle aussi que personne ne peut vivre à notre place. Le devoir de vivre s'est révélé à moi lors de cet après-midi consacré à la chasse. Cet héritage que j'ai reçu est un don de vie qui s'est transformée en une « dette de vie », ce dont je devrai un jour, à mon tour, m'acquitter même si cette dette se présente comme quelque chose d'impossible à payer. Ce que je dois prend la forme du devoir. Cet héritage de mon père, quoiqu'à son insu, devient une promesse qui me lie à mes ancêtres et aux générations futures par la voie de mon fils.

Une autre leçon que l'on peut retirer de ce récit est la nécessité de donner la voix aux malades ainsi qu'à ceux qui les accompagnent, car eux aussi vivent les contrecoups de la maladie. Si le malade meurt, les survivants auront à vivre longtemps avec cette expérience et le sens qu'ils en font. Cette voix peut être à mille lieues du discours médical. En fait, il y a plus dans une expérience de fin de vie que le discours médical ou dans ce que les autres peuvent en dire. Si pour le sociologue Talcott Parsons (7), les malades se caractérisent par un jeu de rôle dans lequel ils acceptent ce que le discours médical attend d'eux, il faudrait maintenant penser que ceux qui les accompagnent sont ceux qui doivent écrire leur propre scénario à partir d'une situation dans laquelle ils se retrouvent dans une sorte de "no man's land", une zone où ils ne sont pas malades ni pour autant totalement biens. Cela exige, en contrepartie, une éthique de l'écoute. Cette expérience montre que nous ne réfléchissons ni ne délibérons selon des principes ou des valeurs, mais selon ce qui nous fait du sens. Et nous avons accès à ce sens qu'en écoutant ce qui cherche à se dire dans les récits de vie sur l'expérience vécue.

C'est bien d'écouter tous ces récits de vie à l'œuvre, mais encore faut-il un lieu qui donne l'occasion de les accueillir. La réception devient la condition de possibilité pour une telle expérience narrative. Cependant, pour accueillir ces récits, il faut d'autres récits. Pour raconter, il faut avoir déjà entendu des récits. Avec la mise en place par tous les moyens techniques à notre disposition, ne sommesnous pas en train d'instaurer une mort inodore? Une mort qui ne serait plus porteuse d'expérience de vie? Une mort qui ne parlerait plus? Le philosophe italien Giorgio Agamben (8), à la suite de Walter Benjamin (9), sonne l'alarme en raison du fait que notre époque était en train de créer un monde appauvri d'expériences, auquel j'ajouterais une société en perte de mémoire. "L'homme moderne rentre chez lui le soir épuisé par un fatras d'événements - divertissants ou ennuyeux, insolites ou ordinaires, agréables ou atroces - sans qu'aucun d'eux se soit mué en expérience » (8). Emmanuel Kant l'avait prédit. Pour lui, il est " possible qu'une foule de phénomènes remplisse notre âme sans qu'il en pût jamais résulter d'expérience »; on assiste "simplement à un jeu aveugle de représentations » (10). Une expérience devient une expérience lorsqu'elle devient mémorable et elle devient mémorable lorsqu'elle renvoie à quelque chose d'autre, souvent invisible et difficile à dire, ce que nous appelons les idées ou le sens des choses.

Si la maladie se caractérise par la perte de sens, on n'a peut-être plus aujourd'hui la culture pour donner un sens à notre vécu. Justement, Benjamin (9) s'exprime ainsi : " L'art de conter est en train de se perdre. II est de plus en plus rare de rencontrer des gens qui sachent raconter une histoire. Et s'il advient qu'en société quelqu'un réclame une histoire, une gêne de plus en plus manifeste se fait sentir dans l'assistance ". II existe une symétrie entre cette perte de narrativité et cette perte de l'expérience de la mort. Dans le même texte, Benjamin fait la remarque suivante : "Aujourd'hui les bourgeois vivent dans des lieux où personne n'est mort [...] et, quand ils arrivent au bout du rouleau, 
sont entassés par leurs héritiers dans des hôpitaux ou des sanatoriums. Or c'est surtout chez le mourant que prend forme communicable non seulement le savoir ou la sagesse d'un homme, mais au premier chef la vie qu'il a vécue, c'est-à-dire la matière dont sont faites les histoires » (9).

Pourtant, on n'a jamais autant parlé de mort. II existe des revues, des commissions d'enquête et des colloques sur la mort. Nous avons des connaissances approfondies sur le deuil, des outils techniques pour soulager la douleur, des soins palliatifs. Ne sommes-nous pas en train de vider cette expérience de son essence? À partir du moment qu'on en discute dans un colloque, souvent avec un souci de scientificité, ne risque-t-on pas de coloniser cette expérience? Certes, en parler, mais à l'aide d'un langage emprunter? C'est toute la question de la vérité qui se pose.

Une dernière leçon, et non pas la moindre, qu'on peut retirer de ce récit de vie est l'importance de la compassion. Une grande compassion m'habite depuis cette expérience de vie. Ici, la compassion doit être distinguée de l'empathie. Comme le souligne la philosophe américaine Martha Nussbaum (11), si l'empathie permet de comprendre le vécu d'autrui, elle ne dit pas comment se conduire moralement, tandis que la compassion, en plus de donner un accès au vécu d'autrui, est accompagnée d'un souci de bien faire. La compassion vient avec un engagement. II n'y a pas de compassion sans solidarité. Le fait que nous sommes les premiers à reconnaître que nous avons été plus que chanceux dans ce moment difficile de vie en raison de l'accès à des soins privilégié ne fait qu'augmenter notre compassion pour ceux qui ont dû vivre des situations semblables et tous ceux qui auront à s'y frotter sans les mêmes conditions. Notre compassion déborde sur un engagement politique. Nous voudrions fortement qu'une expérience comme la nôtre devienne accessible à tous. Cela fait la différence entre un vécu atroce et une expérience de vie qui reconduit à la rencontre de soi.

\section{Encore des imprévus}

Mon père est décédé depuis plusieurs mois. II n'en demeure pas moins que je n'en finis plus de vivre encore des choses inattendues qui me surprennent. Le constat de ce que Monique a pu faire pour mon père afin qu'il puisse vivre une fin de vie respectable en est un exemple. II est fort probable qu'on ne pourra pas lui rendre le change. Ce que Monique a voulu à tout prix éviter à son époux, elle risque de le vivre pour elle-même en raison de la situation géographique de tous ses enfants. Elle risque de croupir dans un centre d'hébergement pour personne âgée. Ceci n'est pas une remarque pour jeter un discrédit sur les centres d'hébergement. Au contraire. II s'agit plutôt de souligner que, peu importe la qualité des soins, rien ne peut se substituer à la qualité relationnelle d'une famille fondée sur l'échange de paroles. Contrairement à ce que l'on peut penser, cela ne nous remplit pas de culpabilité, mais renvoie au sens tragique de la vie. II y a des choses qui nous échappent comme tous ces imprévus que nous pouvons vivre à l'intérieur de soi.

Ce qu'il y a aussi d'imprévu, c'est le souci de débuter, déjà, une oraison funèbre pour Monique afin de lui rendre hommage, de souligner ses qualités ainsi que la différence qu'elle a pu faire dans nos vies. En effet, elle a contribué à l'histoire de nos vies. Cela mérite d'être raconté à son tour. II n'est pas nécessaire d'attendre le décès. Cela permet de mieux comprendre qui est Monique. Cela ne peut qu'engendrer davantage de compassion, surtout que je ne peux pas interférer sur le cours de sa propre vie.

Le plus difficile reste à venir : taire mon expérience. Pourquoi? Pour ne pas blesser les gens qui ont vécu et qui vivront cette épreuve de vie. Aussi, pour ne pas souffrir moi-même d'être incompris. Cela aussi n'était pas prévu. Je l'ai réalisé au moment où j'ai partagé mon expérience avec une autre personne qui vit cette situation : sa mère souffre aussi de la maladie d'Alzheimer. Étant donné que mon père a reçu des soins impeccables de ses proches et d'une équipe spécialisée à la maison, il est permis de croire que mon père a pu conserver une qualité de vie optimale et une certaine lucidité selon les circonstances. Par exemple, il a été en mesure de reconnaître toutes les personnes qui l'entouraient jusqu'au dernier instant, soit avant la sédation. Même s'il ne pouvait pas s'exprimer, il parvenait à nommer ceux qui étaient devant lui par leur prénom, mais sans plus. Intuitivement, nous 
sommes convaincus que si nous avions placé René dans un centre d'hébergement, rapidement, il aurait perdu ses repères jusqu'à perdre la capacité de nous reconnaître. Par conséquent, en comparant leur expérience avec mon propre vécu, les gens commencent à dire qu'il ne s'agissait pas vraiment de la maladie d'Alzheimer. Je vis cela comme une grande violence. Une diminution de mon vécu, voire de mon être. C'est comme ce que nous avions vécu n'avait pas de valeur, ou à tout le moins, possédait moins de valeur. Jorge Semprun (12) s'est exprimé à propos de cette nonreconnaissance de son expérience. Certes, son expérience des camps de concentration et l'expérience de fin de vie de mon père ne sont pas comparables, mais il en demeure que nous sommes confrontés aux mêmes limites du partage. La mort de mon père génère de la peine, mais d'entendre dire que ce n'était pas trop sérieux, induit une souffrance inacceptable. On peut toujours vivre avec une peine, mais jamais avec la souffrance. Comme l'a souligné Levinas (13), la souffrance c'est inutile. Je peux toujours grandir à travers la peine, tandis que la souffrance n'apporte rien, sinon une diminution de mon être. C'est Spinoza qui le dit. Autrement dit, on peut toujours accorder un sens à sa peine, jamais à sa souffrance.

\section{L'ars moriendi}

J'ajouterais que cette expérience a pris sens pour moi parce qu'elle renvoie à de multiples idées. L'une d'elles consiste en une nouvelle compréhension de l'ars moriendi : demeurer ouvert à ce qui apparaît en place de ce qui nous quitte. Mais pour cela, il faut se rendre disponibles, ce qui demande du temps. Une autre expérience est une nouvelle compréhension de ce que je suis, une compréhension rendue possible en raison du récit que j'ai pu faire de cette expérience. Comme le souligne Ricoeur (14-16), c'est dans l'acte de raconter qu'on risque de parvenir à une rencontre de soi, à une lecture de ce que nous avons été, de ce que nous sommes et de ce que nous projetons devenir. Pour cette raison, l'occasion d'écrire ce texte fut l'occasion de préciser ma pensée, de mieux comprendre mon vécu, de comprendre différemment la pensée philosophique et surtout d'offrir une occasion de parler du sens de l'existence.

Cette expérience me conduit à une herméneutique de la philosophie. Nous savons tous que pour Platon, la philosophie consiste à apprendre à mourir (17), alors que pour les stoïciens, elle consiste à accepter la vie, le destin ou l'ordre des choses, et que pour Nietzsche, elle consiste à dire oui à la vie. En fait, pour Platon, à la suite de mon expérience, apprendre à mourir consisterait à apprendre à aimer parce que c'est Éros, le dieu de l'amour, qui conduit les âmes humaines à la vérité, à la reconnaissance de la grande Beauté (18). D'ailleurs, Aristote, dans son Éthique à Nicomaque (5), a mis de l'avant les stratégies que l'être humain doit déployer pour parvenir au bonheur. Si on reprend le titre, Éthique à Nicomaque, il s'agit alors de mener un combat pour la victoire, pour le bonheur. Pour surmonter l'adversité. Autant Platon qu'Aristote nous invitent à vivre l'amour ou le bonheur malgré l'adversité.

Nietzsche va dans le même sens lorsqu'il demande d'aimer la vie, de dire oui à la vie malgré tout. Amor fati. Nous sommes condamnés à aimer malgré tout, malgré nous, malgré les difficultés de la vie. Contrairement aux stoïciens qui demandaient aux hommes de se résigner au destin, Nietzsche invite les hommes à faire un pas supplémentaire, celui d'aimer davantage. Cela implique de cesser d'endurer la vie sans s'y engager. Nietzsche, dans l'un de ses aphorismes dans le Gai savoir (19), indique que l'amour et la beauté, comme chez Platon, sont étroitement liés. Autrement dit, aimer la vie, c'est trouver la vie belle. Cela n'a rien à voir avec le fait de mener la belle vie parce qu'elle est facile.

Les facilités de la vie ne devraient pas servir à nous faire oublier la vie, son sens. Elles devraient être vues comme un terrain sur lequel nous nous exerçons à aimer, pour nous préparer à aimer dans les moments plus difficiles. Dans les Évangiles, il y a cet adage devenu célèbre : tends la joue à celui qui t'a offensé. Tout cela implique que l'amour est de notre ressort, de notre responsabilité et que c'est quelque chose qui s'apprend. C'est tout simple, mais cela demande toute une vie. 
Je termine en rappelant qu'on définit souvent la philosophie comme l'amour de la sagesse. Rarement, on inverse le génitif pour la définir comme la sagesse issue de l'amour.

C'est l'amour de la vie qui nous procure la sagesse, qui nous donne la force d'affronter la vie, qui nous donne les qualités ou les vertus (vertu au sens d'Aristote, comme la tempérance et la prudence) pour surmonter les obstacles de la vie. C'est l'amour qui fait que nous, ses enfants et son épouse, avons pu manifester un souci pour le bien-être de René dès les premiers jours de sa maladie, sans compter un souci pour chacun d'entre nous en respectant nos limites propres, nos situations particulières et nos capacités inégales. Aucune organisation politique des soins de santé ne peut se substituer à ce vécu.

\section{Conclusion}

Que peut-on conclure d'une telle expérience? Si nous pouvons prévoir qu'un jour ou l'autre nous mourrons ou que nous serons témoins de la fin de vie et de la mort d'un autre, il n'en demeure pas moins que l'essentiel se situe là où nous n'avions rien prévu. John Lennon aurait dit que la vie c'est tout ce qui nous arrive pendant que nous sommes occupés à faire autre chose. Ce sont tous ces petits imprévus qui rendent la vie riche d'expériences. II n'était pas prévu que je revisite mon enfance telle que vécue avec mon père, ma mère, mes frères et sœurs, mes cousines et cousins. II n'était pas prévu que je constate la nature temporelle de la famille, ni l'extinction des générations. Plus que tout, il n'était pas prévu qu'un nouveau rapport à soi s'établirait, ni un nouveau rapport à autrui comme un nouveau rapport à la vie. Enfin, il n'était pas prévu que je touche à l'essentiel : faire ce pour quoi je suis fait, c'est-à-dire réfléchir à la vie comme mon père savait si bien le faire. Comme le remarque le théologien et philosophe Paul Tillich (20), il faut tout de même avoir le courage de s'accomplir et, par conséquent, de résister à tous ces obstacles qui nuisent au plein déploiement de notre existence. Pour cela, il faut vivre ses rêves, ses images de soi qu'on porte en soi en dépit des obstacles : c'est l'enjeu éthique par excellence. Le reste a bien peu de valeur. Cependant, ça prend toute une vie pour le comprendre, même si des générations entières se sont souciées de nous transmettre le fruit de leurs expériences, même si nous avons eu tout le support de notre famille, même si nous avons eu une belle enfance. Autrement dit, tout est toujours à recommencer pour chaque individu qui naît et qui prend le relais.

Ainsi, la vie et la mort se nourrissent l'un et l'autre lorsqu'ils se touchent de si près. C'est ce qui arrive dans les actes de soins offerts par les médecins, les infirmières et les aidants naturels. C'est ce qui ressort dans les récits de vie, dans les grandes œuvres littéraires, dans les mythes, avec les symboles, avec les œuvres philosophiques et culturelles. Pour cette raison, la fréquentation des œuvres ne peut que contribuer à la qualité des soins en rappelant le sens profond du soin : faire rencontrer la vie et la mort. À chaque fois que cette rencontre advient, cela nous émerveille et crée un besoin pressant d'exprimer cette expérience aux futures générations pour tracer la voie. En ce sens, les générations passées et les familles deviennent des courroies de transmission qui viennent nourrir ce que nous sommes toute notre vie : des enfants en train d'apprendre à vivre et à aimer la vie et la mort dans l'intimité de notre être.

\section{Références}

1. Descartes, R. 2009. "Discours de la méthode ». CEuvres complètes, vol. III. Paris, Gallimard.

2. Kant, E. 1985. Fondements de la métaphysique des mœurs, dans CEuvres Philosophiques II, Paris, Gallimard.

3. Hadot, P. 2002. Exercices spirituels et philosophie antique. Paris, Albin Michel.

4. Rousseau, J.J. 1966. Émile ou de l'éducation. Paris, Garnier-Flammarion.

5. Aristote. 2004. Éthique à Nicomaque, Paris, GF Flammarion.

6. Manguel, A. 2009. La cité des mots. Arles-Montréal, Actes Sud-Leméac. 
7. Parsons, T. 1951. The Social System. New York, Free Press.

8. Agamben, G. 1989. Enfance et histoire. Dépérissement de l'expérience et origine de l'histoire. Paris, Payot.

9. Benjamin, W. 2000. CEuvres III. Paris, Gallimard.

10. Kant, E. 1944. Critique de la raison pure. Paris, Presses Universitaires de France.

11. Nussbaum, M. 2001. Upheavals of Thought. The Intelligence of Emotions. New York, Cambridge University Press.

12. Semprun, S. 1994. L'écriture ou la vie. Paris, Gallimard.

13. Levinas, E. 1991. Entre-nous. Essais sur le penser-à-l'autre, Paris, Grasset.

14. Ricœur, P. 1983. Temps et récit, Tome I, Paris, Seuil;

15. Ricœur, P. 1985. Temps et récit, Tome III, Paris, Seuil;

16. Ricœur, P. 1990. Soi-même comme un autre, Paris, Seuil.

17. Platon. 2008. «Phedon ». CEuvres complètes, Paris, Flammarion.

18. Platon. 2008. « Le Banquet ». CEuvres complètes, Paris, Flammarion.

19. Nietzsche, F. 1950. Le gai savoir, Paris, Gallimard, coll. " Idées ».

20. Tillich, P. 1999. Le courage d'être. Paris, Cerf. 